\title{
Argumentation-Based Risk Management
}

\author{
Fabrício S. Severo, Ruan C. B. Pozzebon, Lisandra M. Fontoura, Luís A. L. Silva \\ Programa de Pós Graduação em Informática - Universidade Federal de Santa Maria \\ (UFSM) - Santa Maria, RS - Brazil \\ \{severo.fabricio, rb.pozzebon, lisandramf, silva.luisalvaro\}@gmail.com
}

\begin{abstract}
Collaborative risk management is a fundamental task in project management. This paper discusses a new collaborative approach to risk management which is grounded on the integration of dialogue games and argument schemes from argumentation research. In particular, we propose a new dialogue game to organize risk discussions, and the exploitation of typical argument schemes for risk management on the collection of more complete arguments. A new collaborative Risk Discussion System is discussed and evaluated, where its environment is capable of structuring and recording risk discussions in a project management memory.
\end{abstract}

\section{Introduction}

Risk management allows project stakeholders to increase the chances of success of a project and to avoid the surprises that may occur during the project development. However, the lack of exploitation of risk management activities is one of the main causes of unsuccessful projects. According to recognized authors in this area, Boehm (2001) and DeMarco and Lister (2003), effective risk management ought to be developed collaboratively in a task where the viewpoints of different stakeholders are collected and analyzed. Risk management should also exploit techniques that allow such project participants to organize the various kinds of risk information in such a way that this information could be reused later in tasks of risk management of new projects.

Collaborative tasks of risk management were already mentioned by different authors (e.g. Greer and Bustard (2002) and Papadaki et al. (2008)). Despite this interest, little or no research has been made on the identification and modeling of the interaction steps of argumentation that often occur when project stakeholders are involved in tasks of risk management. However, such dialectical interaction activities are likely to contain significant risk management information used by these participants to achieve their conclusions.

In this paper, we present an argumentation-based approach to collaborative risk management. This approach is grounded on the "argumentation" research [Carbogim et al. 2000; Moulin et al. 2002] in which argumentation can be understood as a dialectical process where different users present and analyze arguments in order to find a solution for given problem. In our work, we show that cooperative activities of risk discussion can be organized by means of a new "dialogue game" (DG) [McBurney et al. 2007] for risk management. This DG describes how to organize the exchange of arguments in a risk management debate. Even considering such interaction protocol, however, we observed that these arguments can still be presented as incomplete statements by discussion members. In order to offer these participants ways of advancing well-formed arguments, as well as to reuse arguments that often appear in tasks of risk discussions, 
this paper also discusses the exploitation of a new set of "argument schemes" (AS) [Walton et al. 2008] for risk management. In our work, these schemes are captured as templates for typical risk management arguments that may be reused by users in the development of collaborative risk discussions. In essence, while a DG is able of structuring the exchange of risk management information through the use of an adjusted set of locution acts (or dialogue moves), an AS is able of structuring the reasoning used on the proposition of individual risk discussion statements. Taking these argumentationbased techniques on board, this paper also describes a new web-based system - the Risk Discussion System (RD System v1.0) - which records risk discussions in a reusable project management memory.

The remainder of this paper is organized as follows: Section 2 presents relevant concepts for the understanding of DGs and ASs in the risk management scenario; Section 3 presents our argumentation-based risk management approach; Section 4 discusses experimental results and compares our approach with others; finally, Section 5 presents conclusions.

\section{Background}

Risks are future and uncertain events that could interfere on the correct development of projects. Risk management [Boehm 2001] aims to reduce negative surprises that a project may be exposed during its development. Such risk management practices are directed to the identification of sources of possible future problems in a project, in which project stakeholders can define actions to deal with these sources. In this case, corrective actions can be determined as priorities by project managers.

Certain tasks are common among different risk management frameworks [Georgieva et al. 2009; Stern and Arias 2011]: risk identification, risk analysis, risk planning and risk monitoring and control.

Risk identification is concerned with the construction of a list containing the most likely risks in a project. In this phase, it is important to involve different project stakeholders in a collaborative task which is directed to the analysis of their viewpoints. Risk analysis is concerned with the prioritization of risks that were identified previously, where alternative approaches are available to determine such priorities [Georgieva et al. 2009]. Among these approaches, the criticality of each risk can be considered, which consists of a combination between the probability of a risk occurrence and the impact caused by such occurrence. This phase is useful to avoid additional work on the treatment of risks with low priority. Risk planning is concerned with the elaboration of plans aiming to avoid that prioritized risks occur. Planning is also concerned with the reduction of the negative impact due to the occurrence of risks. Plans may also contain a description of actions which should be introduced on the process of project development. Finally, risk monitoring and control are concerned with the observation of prioritized risks and application of contingency plans whenever they are necessary. In this phase, new risks may be discovered and prioritized. This implies that risk management is an iterative activity during the project development.

Different authors (e.g., Boehm (2001) and DeMarco \& Lister (2003)) claim that risk management should be a cooperative task involving most project stakeholders. In our work, collaborative tasks are modeled as a process of "argumentation". Argumentation studies the structure of arguments and the process of arguing [Carbogim 
et al. 2000; Moulin et al. 2002]. An argument is a claim that one believes it is true. The main characteristic of arguments is the fact that an argument may or may not be true in a point in time. Arguing (or argumentation) is the act of using arguments to explain or justify a point of view. Computational argumentation, consequently, involves the use of computers for constructing, recording and using arguments. Despite the fact that argumentation is already being exploited in medical decision support systems, legal systems and agent communication, for instance, there is little or no research on the exploitation of argumentation techniques on the development of risk management tasks.

In our work, the collaborative discussion of risks is modeled as a dialogue in which project participants work together so that they could find solutions (plans) for a problem (risks). In argumentation research, the notion of "dialogue games" [McBurney et al. 2007] can capture such kind of dialogue among project participants. A dialogue game represents participants' actions in a conversation by means of speech moves (e.g., to ask, to inform, etc.). The main structures of a DG are: (1) rules determining when and how a dialogue should start and finish; (2) a set of actions (locutions or speech acts) representing the allowed interactions among dialogue participants; (3) combination rules defining how locutions should interact each other, i.e. under which circumstances it is possible to use a certain locution as a response to a speech move advanced in the debate.

In a conversation which is mediated by our dialogue game protocol, participants select and use speech acts along with individual statements (i.e. pieces of textual information). These are statements representing the content of the speech message (e.g., what one is asking, what one is informing, etc). For instance, we can analyze a speech act presented in the example of Section 3.1: "Inform: The programmers don't have the technical skills in hardware programming and they don't have knowledge in the selected algorithms". Notice that the Inform speech act amounts to a dialogue-based indexing structure for these textual claims. In fact, the Inform locution move represents explicitly alternative steps of interaction (or locutions) between dialogue participants. However, this example also shows that one should have ways of collecting individual risk management statements, which are particular arguments presented by users in the overall process of conversation. In general, these arguments ought to be well-formed and structured (which is not the case, in many situations), containing risk discussion claims that are constructed and presented clearly so that debate participants could understand them. In argumentation research, the capture, organization and reuse of such kind of well-formed arguments can be related to the notion of "argument schemes" (AS) [Walton et al. 2008].

An AS aims to represent the key elements of stereotypical patterns of arguments. As Table 1 shows, they are commonly represented in terms of premises and conclusion. In addition, the representation of schemes requires the definition of a set of "critical questions" (CQs). In essence, these questions can be used by users to determine whether an argument is fallacious or not (the act of determining the validity of an argument). The act of answering these critical questions can also help debate participants to continue a discussion in meaningful ways. Once a critical question is selected, for instance, one can present a further argument to question the truth of a statement which was advanced according to a particular scheme. One can also present new statements to support (to back up) a scheme-based argument that was advanced previously in a 
discussion. A rather classical example of these schemes is the argument from expert opinion (Table 1) presented by Walton et al. (2008).

Table 1. Argument from expert opinion

\begin{tabular}{|c|l|}
\hline Major Premise & Source E is an expert in subject domain D containing proposition A \\
\hline \multirow{2}{*}{ Minor Premise } & E asserts that proposition A (in domain D) is true (false) \\
\hline \multirow{5}{*}{ Conclusion } & A may plausible be taken to be true (false) \\
\hline \multirow{4}{*}{ CQitical Questions } & CQ2. How credible is E as an expert source? \\
\cline { 2 - 3 } & CQ3. What did E assert that implies A? \\
\cline { 2 - 3 } & CQ4. Is E personally reliable as a source? \\
\cline { 2 - 3 } & CQ5. Is A consistent with what other experts assert? \\
\cline { 2 - 3 } & CQ6. Is E's assertion based on evidence? \\
\hline
\end{tabular}

\section{The Argumentation-Based Approach to Collaborative Risk Management}

The key components of our argumentation-based approach to collaborative risk management are i) a new dialogue game which organizes the interactions between discussion participants and ii) the exploitation of argument schemes for risk management on the collection of well-formed statements.

\subsection{A Dialogue Game for Risk Management}

The new DG to support the development of the collaborative tasks of risk management is formed by a set of locution acts along with rules describing how these locutions can be combined (i.e. locutions used as responses to previously advanced speech acts). The key idea of our DG is to exploit the integration of standard locution acts (as presented by McBurney et al. (2007), for instance) with tasks of risk identification, analysis and planning. In our opinion, those are the tasks that mostly require the use of a discussion protocol (while monitoring and control, not yet treated in our protocol, involve the data collection and application of pre-defined plans). The key elements of this DG are presented in the Table 2. In order to understand how a dialogue game mediates a collaborative task of risk management, Figure 1 presents an example of a risk discussion (this sample discussion is limited to a single risk due to limitations of space). In this example, risk identification speech moves are mainly advanced through the use of Propose Risk locution act. Risk analysis is developed by means of Propose Probability and Propose Impact. Risk planning is captured by the Propose Plan locution act.

According to our DG protocol, a discussion starts when a Start Discussion locution is advanced. The next step in the discussion is the proposition of risks. In the example, the risk stated was that "The project members don't have the technical skills required for the development of this project". The probability and impact of each risk that is proposed by participants are analyzed. Risks with higher probability/impact are selected by means of the Select locution act. Risks that are selected are usually managed by risk contingency or mitigation plans, among others kinds of plans. In the discussion, the Propose plan is used to describe the overall content of a plan. Once multiples plans can be advanced, participants can also be involved in the selection of the plans that will be applied in the project. As this example shows, other locutions can be exploited 
during the discussion in order to capture and represent the alternative opinions regarding the risks being assessed in the project.

Table 2. A dialogue game protocol for risk management

\begin{tabular}{|c|c|c|}
\hline Locution & Use & Response \\
\hline Start Discussion & $\begin{array}{l}\text { Starting the dialogue, giving information about what has to } \\
\text { be done }\end{array}$ & $\begin{array}{l}\text { Ask; Inform; Propose Risk; } \\
\text { Summarize; Ask Position; End } \\
\text { Discussion }\end{array}$ \\
\hline Propose Risk & $\begin{array}{l}\text { Making propositions of risks that shall be discussed. This is } \\
\text { the central element of the discussion, where all other } \\
\text { locutions will focus }\end{array}$ & $\begin{array}{l}\text { Ask; Inform; Propose } \\
\text { Probability; Propose Impact; } \\
\text { Propose Plan; Propose } \\
\text { Consequence; Argument-pro; } \\
\text { Argument-con; Withdraw; } \\
\text { Summarize; Ask Position; } \\
\text { Select }\end{array}$ \\
\hline $\begin{array}{l}\text { Propose } \\
\text { Probability }\end{array}$ & $\begin{array}{l}\text { Used along with a Propose Risk in order to state a risk } \\
\text { probability. This probability can be expressed either } \\
\text { qualitatively (e.g., low, medium or high) or quantitatively } \\
\text { (e.g., } 10 \%, 75 \% \text { ), or both }\end{array}$ & $\begin{array}{l}\text { Ask; Inform; Argument-pro; } \\
\text { Argument-con; Withdraw; Ask } \\
\text { Position; Select }\end{array}$ \\
\hline Propose Impact & $\begin{array}{l}\text { Used along with a Propose Risk so that a risk impact } \\
\text { proposition could be stated. This impact can be expressed } \\
\text { either qualitatively or quantitatively (e.g., in terms of money } \\
\text { or time spent), or both }\end{array}$ & $\begin{array}{l}\text { Ask; Inform; Argument-pro; } \\
\text { Argument-con; Withdraw; Ask } \\
\text { Position; Select }\end{array}$ \\
\hline Propose Plan & $\begin{array}{l}\text { Describes possible management plans to deal with the risk. } \\
\text { Those plans should contain the description of actions and } \\
\text { tasks that can be executed during the development of the } \\
\text { project. The proposition of plans aim to prevented, eliminated } \\
\text { or reduced risks }\end{array}$ & $\begin{array}{l}\text { Ask; Inform; Propose } \\
\text { Consequence; Argument-pro; } \\
\text { Argument-con; Withdraw; Ask } \\
\text { Position; Select }\end{array}$ \\
\hline $\begin{array}{l}\text { Propose } \\
\text { Consequence }\end{array}$ & $\begin{array}{l}\text { Used by users when assessing consequences related to the } \\
\text { occurrence of a risk or the application of a risk management } \\
\text { plan, for instance }\end{array}$ & $\begin{array}{l}\text { Ask; inform; Argument-pro; } \\
\text { Argument-con; Summarize }\end{array}$ \\
\hline Select & $\begin{array}{l}\text { Choosing of a probability and an impact during risk } \\
\text { prioritization tasks, selecting a risk to be considered in the } \\
\text { planning phase and choosing a Risk Management plan to be } \\
\text { applied in the project. This locution is used to make explicit } \\
\text { all the choices made during the risk discussion }\end{array}$ & Withdraw \\
\hline Withdraw & $\begin{array}{l}\text { Withdrawing (i.e. giving up) from claims presented in the } \\
\text { dialogue. This move tells other participants that one no } \\
\text { longer agrees with a previous risk assessment statement }\end{array}$ & No response needed \\
\hline Argument-pro & $\begin{array}{l}\text { Advancing an argument in favor of a claim, or presenting } \\
\text { information that gives support to such risk discussion claim }\end{array}$ & $\begin{array}{l}\text { Ask; Inform; Argument-pro; } \\
\text { Argument-con }\end{array}$ \\
\hline Argument-con & Advancing an argument against a claim, or rebutting it & $\begin{array}{l}\text { Ask; Inform; Argument-pro; } \\
\text { Argument-con }\end{array}$ \\
\hline Ask & $\begin{array}{l}\text { Asking questions to other participants. Questions can involve } \\
\text { any subject that is relevant to the risk assessment dialogue }\end{array}$ & Inform; Summarize \\
\hline Inform & $\begin{array}{l}\text { Advancing information to better understand the risk } \\
\text { discussion or to answer a question which is advanced } \\
\text { previously }\end{array}$ & $\begin{array}{l}\text { Ask; Inform; Argument-pro; } \\
\text { Argument-con; Summarize }\end{array}$ \\
\hline Summarize & $\begin{array}{l}\text { Summarizing pieces of information from the discussion in a } \\
\text { single statement. This locution makes easier the finding of } \\
\text { relevant information in the dialogue }\end{array}$ & No response needed \\
\hline Ask Position & $\begin{array}{l}\text { Asking the opinion of other participants in face of } \\
\text { controversial statements presented }\end{array}$ & Opinion \\
\hline Opinion & $\begin{array}{l}\text { Expressing an opinion in order to answer a request which is } \\
\text { emitted when the Ask Position locution is used }\end{array}$ & No response needed \\
\hline End Discussion & $\begin{array}{l}\text { Closing the dialogue; once the discussion is ended, new } \\
\text { arguments cannot be advanced }\end{array}$ & No response needed. \\
\hline
\end{tabular}




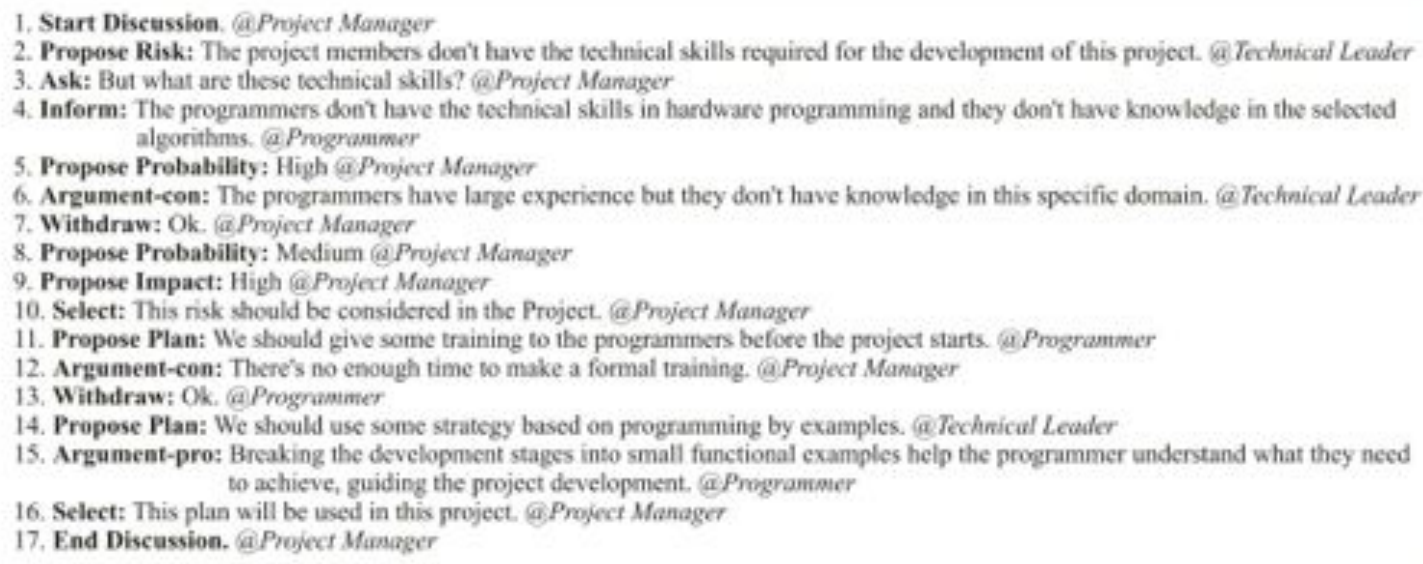

Figure 1. An example of our DG protocol in practice

\subsection{Argument Schemes for Risk Management}

In risk management discussions, additional information regarding project risks are frequently requested by project participants. As the example in Figure 1 shows, additional risk information was requested by the Project Manager - see the (Ask \#3) speech move. Requests for more information can be due to different reasons. For instance, a discussion participant may need additional information because a certain statement was not proposed in a comprehensible form. In effect, the dialogue game protocol is useful to keep the coherence among locutions acts used during the discussion. However, these locutions are still advanced by users along with/connected to free-text statements. Therefore, one still needs ways of collecting, formalizing and reusing the key parts of these statements. As proposed in this paper, this is developed through the exploitation of "argument schemes".

ASs help users when presenting a structured contextualization for their arguments (i.e., premises that lead to the conclusion). This structure may also prevent discussion participants to advance incomplete and inconsistent arguments. Moreover, the set of critical questions provide users with clues (sometimes understood as checklists directed to the analysis of an argument) for the validation and expansion of the risk management debate. For instance, an argument scheme used when proposing a risk regarding the lack of experience of a project participant may have a critical question "Can the programmers be given training on the technology?". In this case, the answer of this critical question can lead to the proposition of a plan to deal with this risk: "Giving training on the technology may reduce this risk".

In our project, we constructed a library of argument schemes for risk management. In order to identify and represent such schemes, we took advantage of the notion of "argument types" (AT) [Silva et al. 2010]. An AT aims to capture the nature of an argument in the application domain (i.e. what the argument is about). In our work, these types describe what an argument is about in the risk management scenario. This means that users can link their ASs with one or various types. That is because risk management statements can be constructed through the combination of different subjects in the application domain. Although the notion of ASs offers a representation for well-formed argument components, we observed that the use of ATs during the process of AS design tends to be simpler for ordinary users in an application domain. In effect, a list of such types can already be available in the application, as lists of other 
kinds of types (e.g., risk types) are available on the project management literature [Sommerville 2011], for instance. Taking advantage of such risk categorization lists (e.g., Sommerville (2011)), the key ATs defined in our project are: AT01 - Client; AT02 - Requirements; AT03 - Planning; AT04 - Execution; AT05 - Maintenance; AT06 Business; AT07 - Technology; AT08 - Organizational; e AT09 - Tools. In our project, we observed that this simple list of ATs is enough to index the most relevant arguments presented by project stakeholders in risk management tasks.

We can present a sample of risk management argument scheme developed in our project. One of these schemes is relevant to the analysis of risks related to the lack of participants' skills in a project (Table 3). This scheme is indexed by the "Execution" and "Technology" ATs. In Figure 1, we presented an example of this kind of argument.

Table 3. An argument scheme for risk management

\begin{tabular}{|l|l|}
\hline \multirow{2}{*}{ Scheme: Risk Management argument scheme from lack of skills } \\
\hline \multirow{2}{*}{ Conclusion } & Therefore, there is a lack of necessary technical skills in $\mathrm{Z}$. \\
\cline { 2 - 2 } & 2. It is not possible to recruit Y with technical skills in Z. \\
\hline \multirow{2}{*}{$\begin{array}{l}\text { Critical } \\
\text { Questions }\end{array}$} & CQ1. Does $\mathrm{X}$ really need to be based on $\mathrm{Z}$ ? \\
\cline { 2 - 3 } & CQ2. Can $\mathrm{Y}$ be given training on $\mathrm{Z}$ ? \\
\cline { 2 - 3 } & $\mathrm{CQ3}$. Do all Y need to have technical skills in Z? \\
\hline
\end{tabular}

Table 3 shows that AS components can be formed by variables $\mathrm{X}, \mathrm{Y}$ and $\mathrm{Z}$, where such variables can be instantiated by different terms in a risk management discussion (e.g., assuming $\mathrm{X}$ as a project, $\mathrm{Y}$ as a team member or a group of members, and $\mathrm{Z}$ as a technology used in the project). Table 4 shows an instantiation of this argument scheme (as presented in Figure 1).

Table 4. An instantiation of an argument scheme for risk management

\begin{tabular}{|l|l|}
\hline \multirow{5}{*}{ Premises } & $\begin{array}{l}\text { 1. The project requires that programmers have technical skills in hardware } \\
\text { programming. }\end{array}$ \\
\cline { 2 - 3 } & $\begin{array}{l}\text { 2. It is not possible to recruit programmers with technical skills in hardware } \\
\text { programming. }\end{array}$ \\
\hline \multirow{2}{*}{$\begin{array}{l}\text { Conclusion } \\
\text { Qritical }\end{array}$} & Therefore, there is a lack of necessary technical skills in hardware programming. \\
\hline & CQ1. Does the project really need to be based on hardware programming? \\
\cline { 2 - 3 } & CQ2. Can programmers be given training on hardware programming? \\
\cline { 2 - 2 } & CQ3. Do all programmers need to have technical skills in hardware programming? \\
\hline
\end{tabular}

Due to the exploitation of typical ASs, information required to better understand a proposed risk is presented explicitly during the debate. Once discussion participants select a scheme, they can examine their critical questions before or after the instantiation of the scheme (in case a scheme is selected from a scheme library, or a scheme is selected from a past discussion recorded in a risk management memory). This analysis may result in a stronger argument, or even in counter-arguments that could defeat an argument already advanced in the risk management debate. As the first critical 
question in Table 3 shows, for example, an instance of that AS (Table 4) could be defeated "if the project could be based on a technology that is different from hardware programming". Moreover, critical questions can lead users to the declaration of possible plans to deal with risks in the project. For example, the second critical question in Table 3 shows that a training plan on hardware programming could be offered to project members. As Figure 1 describes, this plan was advanced by a discussion participant indeed (see speech act \#11). However, due to lack of available time to conclude the project, the plan that ended being selected by discussion members involved the use of both hardware programming examples and the continuous support during the project development on the informal training of project participants.

\subsection{The Risk Discussion System}

In our project, we developed a web-based collaborative system called Risk Discussion System (RD System). The RD System is fully responsible for the integration of our dialogue game and our argument schemes for risk management during the development of collaborative risk discussions.

Risk discussions are managed in the system as activities of a project. Each project is defined by a set of factual characteristics. In the system, users are able to define and adjust these characteristics to the needs of an organization. Figure 2 presents the main components of the RD System. The architecture involves a risk discussion user interface, while the risk discussion engine controls the communication between the participants. Importantly, the RD System keeps records of all steps of collaborative risk discussion, allowing users to form a structured project management memory.

According to such architecture, the elements of the DG protocol are represented in an external XML file. Once this protocol is loaded in the system, the protocol elements (locutions and rules) are interpreted by the RD System automatically. In the XML representation, protocol elements are represented by tags such as $<$ locutions $>$ and $<$ responses $>$, for instance. The overall idea of such external protocol representation is the possible need of protocol exploitation in different risk management systems.

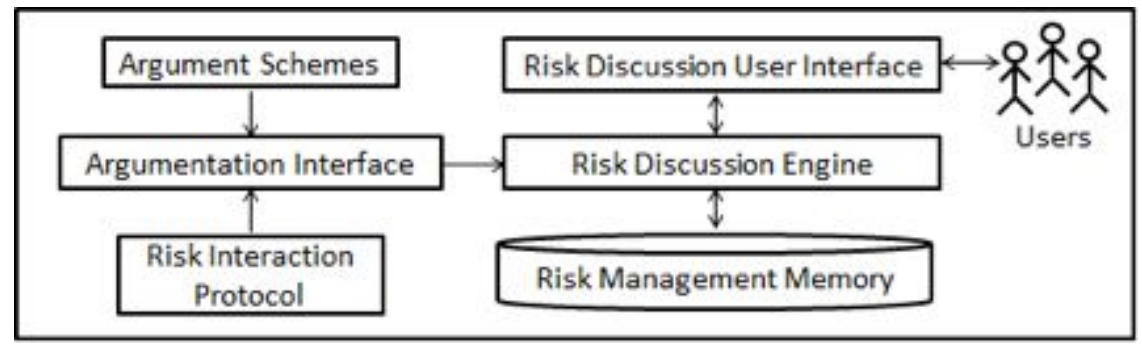

Figure 2. The RD System architecture

\subsubsection{The Use of the Dialogue Game for Risk Management}

The RD System presents the dialogue game-based discussions in a tree-based format. As Figure 3 shows, each locution act corresponds to a node in this discussion tree. Users are able to inspect such tree-based representation, as well as to filter the discussion branches that are deemed relevant to the analysis of their risk management issues. The addition of new locutions in such tree is done when users select the tree node where both the new locution and its textual content should be inserted. Then, a new locution act is inserted as a children node of that selected node. This also means that when a 
certain node in the tree is selected by users, the system controls what responses can be inserted along with the selected speech act. This control indicates that the combination rules of the interaction protocol are managed by the RD System automatically.

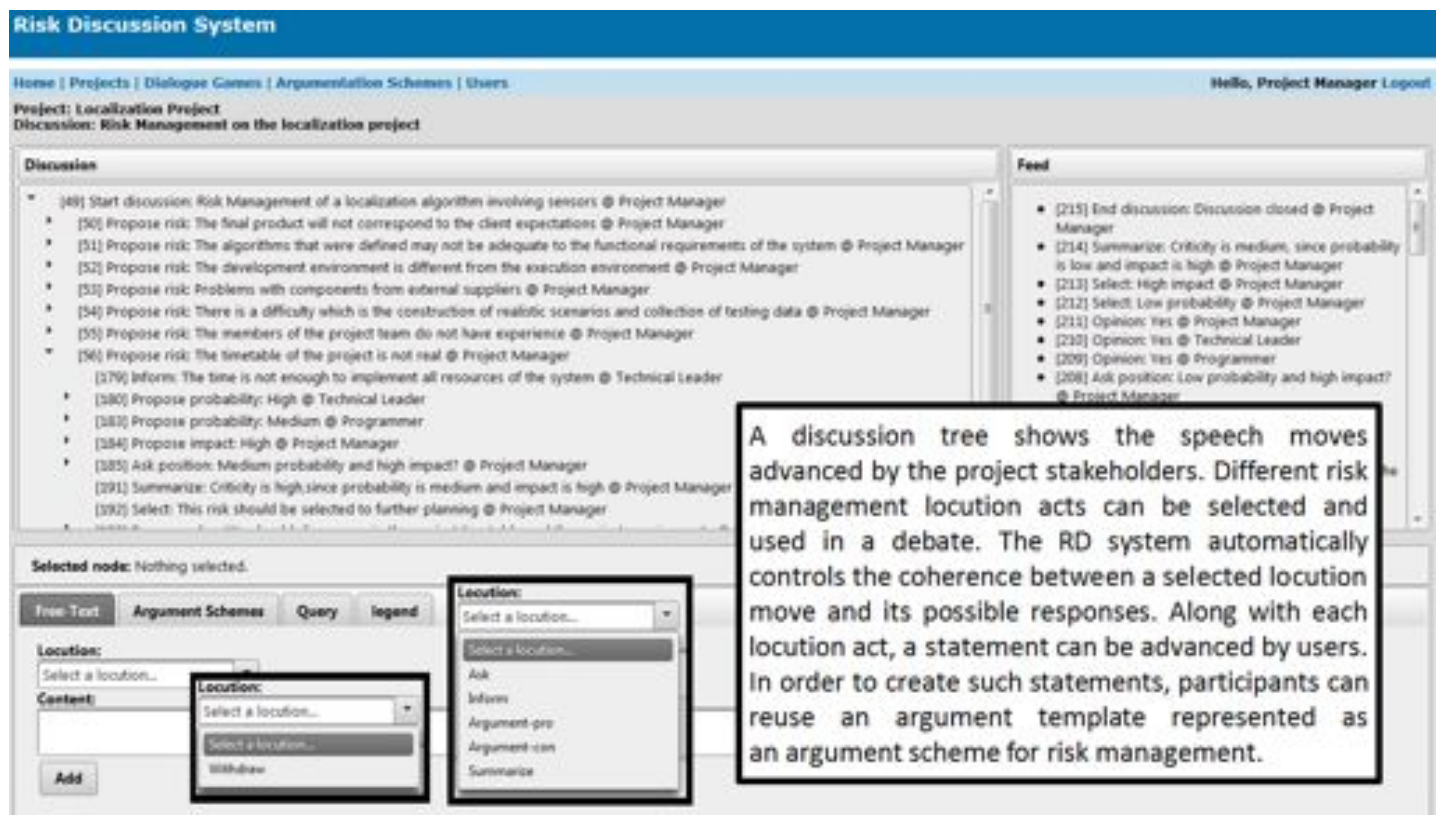

Figure 3. The collaborative discussion resources of the RD System

\subsubsection{The Use of Argument Schemes for Risk Management}

The RD System is able to manage argument schemes. In our approach, an AS should be indexed by one or more argument types - what the argument is about in the application domain (AT01 - Client; AT02 - Requirements, etc). Those types act as indexing structures to filter ASs recorded in the system's memory. This filtering allows users to retrieve and reuse such schemes in risk management discussions.

The recording of AS and ATs in the RD System can be made in two ways. The first way is using the RD System to type argument types along with scheme components (premises, conclusion and critical questions). The system presents an interface where users can fill forms leading to the registration of such prototypical arguments. Another form of adding new schemes on the system is by importing a scheme library (as a library of argument schemes presented by others, e.g. the Walton library [Walton et al. 2008] - a set of generic argument schemes, but still useful ones in the development of risk management tasks). However, this library should be described in a XML-based argument scheme representation, following an AML notation [Reed and Rowe 2004].

In a collaborative risk management discussion, users can make use of the schemes along with the speech acts defined in our DG, as Figure 4 shows. In a discussion recorded in the system's memory, users can easily identify arguments that were advanced according to a predefined AS (instead of arguments described in terms of free-text statements only). When an argument is an instance of a certain scheme, a scheme icon " $\mathrm{S}$ " is presented along with a statement in the discussion tree. Through this icon, any discussion participant is able to inspect the entire scheme structure used there in the debate and possibly answers for its critical questions. 


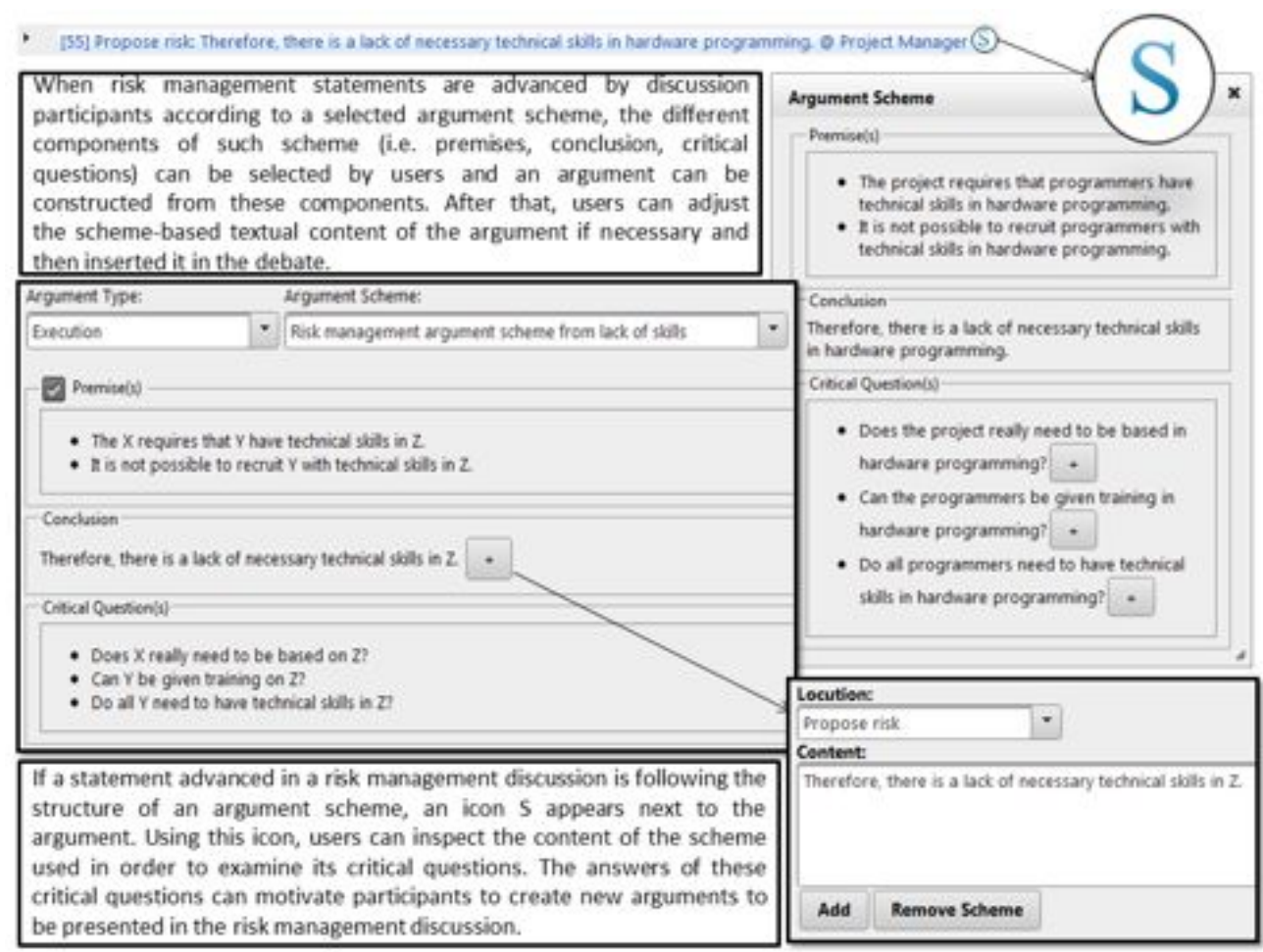

Figure 4. The argument scheme resources of the RD System

\section{Evaluation and Discussion}

In order to evaluate the relevance of the approach proposed in this paper (and its concrete representation in the RD System), four experiments were developed, consisting in the use of the RD System on the development of risk management activities of a fictitious project. Based on participant questionaries' answers (16 participants divided in distinct discussion groups), we can point out the following results: (a) 94\% strongly agreed that it is important to exploit collaboration activities in order to reach a good risk management process; (b) $81 \%$ strongly agreed and 19\% agreed that it is important to record risk management discussions; (c) 63\% strongly agreed and 38\% agreed that the proposed dialogue game is capable of expressing the most important aspects in a risk management debate; (d) $44 \%$ strongly agreed and 56\% agreed that the proposed dialogue game organizes risk discussions properly; (e) $81 \%$ strongly agreed and 19\% agreed that having the support of the RD System is important to achieve a good risk management process; and (f) $25 \%$ strongly agreed and $75 \%$ agreed that with the RD System allows the identification of what is already complete and what still needs to be achieved when tasks of risk management are being developed.

Argumentation systems in which multiple users can interact with each other in order to search decisions for controversial subjects were proposed in the past in other application domains. Among others, we can point out the HERMES [Karacapilidis and Koukouras 2006] and Carrel+ [Tolchinsky et al. 2006] systems.

In the HERMES system, argumentation is developed through a model which is inspired on the IBIS model [Shum et al. 2006]. This system is intended to provide computer-supported argumentation capabilities for group decision-making problems. In the HERMES model, users involved in a collaborative process of discussion or negotiation make their "viewpoints" - a concept representing a user's general interests 
and goals - transparent to the others. In HERMES, argumentation is performed by "discourse acts" aimed at challenging an opponent's opinion. The argumentation framework is a semi-formal representation expressed in terms of issues, alternatives, positions, pro and con arguments and preferences. In effect, these representation elements may be related to argumentation frameworks for the capture of decision rationales - the IBIS model. Different from the HERMES model, in which the challenging discourse moves are central, our argumentation approach is focused on the representation of collaborative interaction moves for the deliberation of risk management issues. It means that deliberation of risk characteristics (issues, alternatives, etc) are captured along with speech acts for risk management (i.e. a protocol for collaborative risk management).

In the Carrel+ system, discussions of transplant decisions were developed by means of a set of application-oriented ASs. These schemes are motivated on the formalization of the knowledge which is contained in transplant guidelines. Such transplant guidelines (or rules) are often well-defined in this application domain. Hence, decisions should follow those transplant rules. Similarly, their argumentation model shows that debate of transplant decisions is fully developed by pro and con arguments which are constructed according to the transplant guideline schemes available. Different from this model, there is no standard set of guidelines for the risk management, which limits the development of a full set of schemes in this application domain. In many projects, for instance, the risk management is developed informally by users. Although it is important to capture more complete arguments in risk management debates, we believe that we still need to allow users to present arguments just as statements.

\section{Conclusion}

This paper presents a new collaborative approach, grounded on argumentation, for risk management aiming to promote the recording of risk discussions in a memory. The approach uses dialogue games for structuring risk discussions; and argument schemes for the users' proposition of well-formed statements. In addition, a web-based Risk Discussion system was implemented and tested, allowing multiple users to collaboratively discuss the risks of a project.

The use of this approach has benefits to the achievement of effective risk management. Among others, the exploitation of both dialogue games and argument schemes amount to a principled argumentation approach for the structuring and formalization of risk discussions (instead of relying on chat resources only, for instance). Moreover, through the integration of dialogue games with argument schemes, the approach is able to promote the collection and recording of risk statements presented along with speech acts exploited on the development of collaborative risk management debates. At the same time, these argumentation techniques can be used on the systematic construction of an argumentation-based memory which is relevant to support the development of risk management tasks in different projects of an organization.

Future works involve the possibility of extending this approach to the development of tasks of risk monitoring and control; the development of additional experiments to further validate the proposed approach; and the design of argumentationbased query methods aiming to promote the reuse of risk management discussion information recorded in the project management memory. 


\section{References}

Boehm, B. W. (2001). Software Risk Management: Principles and Practices. IEEE Software, 32-41.

Carbogim, D. V., Rovertson, D., Lee, J. (2000). Argument-Based Applications to Knowledge Engineering. The Knowledge Engineering Review, 119-149.

DeMarco, T., Lister, T. (2003). Waltzing with Bears: Managing Risks on Software Projects. Dorset House Publishing Co., Inc.

Georgieva, K., Farooq, A., Dumke, R. R. (2009). Analysis of the Risk Assessment Methods-A Survey. Proceedings of the International Conferences on Software Process and Product Measurement, pp. 76-86.

Greer, D., Bustard, D. W. (2002). Collaborative Risk Management. Systems, Man and Cybernetics, 2002 IEEE International Conference on.

Karacapilidis, N., Koukouras, D. (2006). A Web-Based System for Supporting Collaboration Towards Resolving Oncology Issues. Oncology reports, 11011107.

McBurney, P., Hitchcock, D., Parsons, S. (2007). The Eightfold Way of Deliberation Dialogue. International Journal of Intelligent Systems, 96-132.

Moulin, B., Irandoust, H., Bélanger, M., Desbordes, G. (2002). Explanation and Argumentation Capabilities: Towards the Creation of More Persuasive Agents. Artificial Intelligence Review, 169-222.

Papadaki, E., Polemi, D., Damilos, D. K. (2008). A Holistic, Collaborative, Knowledgesharing Approach for Information Security Risk Management. The Third International Conference on Internet Monitoring and Protection, pp. 125-130.

Reed, C., Rowe, G. (2004). Araucaria: Software for Argument Analysis, Diagramming and Representation. Journal on Artificial Intelligence Tools, pp. 961-979.

Shum, S. J., Selvin, A. M., Sierhuis, M., Conklin, J., Haley, C. B., Nuseibeh, B. (2006). 15 Years on from gIBIS and QOC. In: A. H. Dutoit, R. McCall, I. Mistrik, \& B. Paech, Hypermedia Support for Argumentation-Based Rationale (pp. 111-132). Springer.

Silva, L. A., Campbell, J. A., Eastaugh, N., Buxton, B. F. (2010). A Case for Folk Arguments in Case-Based Reasoning. Proceedings of the 18th International Conference on Case-Based Reasoning Research and Development, pp. 317-331.

Sommerville, I. (2011). Engenharia de Software 9a edição. São Paulo: Pearson.

Stern, R., Arias, J. C. (2011). Review of Risk Management Methods. Business Intelligence Journal, pp. 59-78.

Tolchinsky, P., Cortés, U., Modgil, S., Caballero, F., López-Navidad, A. (2006). Increasing Human-Organ Transplant Availability: Argumentation-Based Agent Deliberation. IEEE Intelligent Systems, pp. 29-37.

Walton, D., Reed, C., Macagno, F. (2008). Argumentation Schemes. New York: Cambridge University Press. 